\title{
Highly efficient CRISPR/HDR-mediated knock-in for mouse embryonic stem cells and zygotes
}

\author{
Bangmei Wang ${ }^{1,{ }^{*}}$, Kunyu Li ${ }^{1,{ }^{*}}$, Amy Wang ${ }^{1}$, Michelle Reiser ${ }^{1}$, Thom Saunders ${ }^{2}$, Richard F Lockey ${ }^{1,3}$, \\ and Jia-Wang Wang ${ }^{1}$ \\ ${ }^{1}$ Division of Allergy and Immunology, Department of Internal Medicine, University of South Florida Morsani \\ College of Medicine, Tampa, FL, 'University of Michigan, Ann Arbor, MI, and 'James A. Haley Veterans' \\ Hospital, Tampa, FL \\ ${ }^{*}$ B.W. and K.L. contributed equally to this work
}

BioTechniques 59:201-208 (October 2015) doi 10.2144/000114339

Keywords: CRISPR cleavage; double-strand break (DSB); homology-directed-repair (HDR); embryonic stem (ES) cells; zygote

Supplementary material for this article is available at www.BioTechniques.com/article/114339.

The clustered regularly interspaced short palindromic repeat (CRISPR) gene editing technique, based on the non-homologous end-joining (NHEJ) repair pathway, has been used to generate gene knock-outs with variable sizes of small insertion/deletions with high efficiency. More precise genome editing, either the insertion or deletion of a desired fragment, can be done by combining the homology-directed-repair (HDR) pathway with CRISPR cleavage. However, HDR-mediated gene knock-in experiments are typically inefficient, and there have been no reports of successful gene knock-in with DNA fragments larger than $4 \mathrm{~kb}$. Here, we describe the targeted insertion of large DNA fragments (7.4 and $5.8 \mathrm{~kb})$ into the genomes of mouse embryonic stem (ES) cells and zygotes, respectively, using the CRISPR/HDR technique without NHEJ inhibitors. Our data show that CRISPR/HDR without NHEJ inhibitors can result in highly efficient gene knock-in, equivalent to CRISPR/HDR with NHEJ inhibitors. Although NHEJ is the dominant repair pathway associated with CRISPR-mediated double-strand breaks (DSBs), and biallelic gene knock-ins are common, NHEJ and biallelic gene knock-ins were not detected. Our results demonstrate that efficient targeted insertion of large DNA fragments without NHEJ inhibitors is possible, a result that should stimulate interest in understanding the mechanisms of high efficiency CRISPR targeting in general.

Genome editing is a powerful tool to study gene function. The $\underline{\text { clustered }}$ regularly interspaced short palindromic repeat (CRISPR) system is an adaptive immune system found in bacteria. It can destroy naturally occurring and engineered phages and plasmids (1). A CRISPR genome editing tool was developed based on this system and has been used to edit the genomes of many species (2-4). CRISPR cleavage causes DNA double-strand breaks (DSBs), which are usually repaired by the nonhomologous end-joining (NHEJ) repair pathway
$(5,6)$. NHEJ is an error-prone process that often causes insertion/deletion (indel) mutations, a portion of which result in frameshift mutations. In one study, a 51\%-79\% CRISPR-targeting efficiency was obtained for different genes in human embryonic stem (ES) cells. Similarly, CRISPR-induced mutation rates of up to $78 \%$ were obtained in mice $(4,8-10)$.

For precise genome editing by inserting or deleting a designed fragment, gene targeting based on homologous recombination $(\mathrm{HR})$ is often the preferred methodology. However, the efficiency of traditional gene targeting is generally low, ranging from undetectable to $0.1 \%$ (11). The DSBs induced by CRISPR can also be repaired through the homologydirected-repair (HDR) pathway. Here, a DNA fragment flanked by two sequences that are homologous to the sequences flanking the cleaved site can be inserted into the cleaved site by HR with efficiencies 5000 times higher than traditional HR (12).

Gene knock-in techniques mediated by the CRISPR/HDR pathway have been less well studied, but CRISPR/HDR

\section{METHOD SUMMARY}

Here we show that large DNA fragments (up to $7.4 \mathrm{~kb}$ ) can be inserted into the genome of mouse embryonic stem (ES) cells at high efficiency using the CRISPR/homology-directed-repair (HDR) technique without the need for non-homologous end-joining (NHEJ) inhibitors. 
targeting efficiencies have been reported to be only in the range of $0.5-20 \%$, much lower than the efficiency of CRISPR mediated by NHEJ $(4,8)$. Since HDR and NHEJ compete with each other for the same DSB site, the efficiency of HDR can be significantly increased by inhibiting NHEJ $(13,14)$. However, because NHEJ is essential for DNA repair and embryonic viability, its deficiency may cause deleterious effects, such as mutagenesis and toxicity. Furthermore, it is unknown whether or not inhibiting NHEJ is effective for knocking in a DNA fragment at any locus, especially large fragments (>4 kb) containing multiple genes or a single large gene. So far, only small inserts and a few loci have been tested with this method $(13,14)$. More importantly, the targeting efficiency of CRISPR in vivo is only $3 \%$ (15). Therefore, additional investigation is required to enhance gene knock-in efficiencies for larger DNA fragments.

Here, large DNA fragments, 7.4 and $5.8 \mathrm{~kb}$, were knocked in to the genomes of mouse ES cells and zygotes, respectively, using the CRISPR/HDR technique without any NHEJ inhibitors, at high efficiencies equivalent to that of CRISPR/ NHEJ knockout in human ES cells (7) and to that of CRISPR/HDR using NHEJ inhibitors $(13,14)$. Furthermore, a high survival rate of zygotes was obtained by injecting only one fourth of the regular concentrations of CRISPR reagents. This study also demonstrates that NHEJ inhibitors and homologous sequences longer than 2.2 $\mathrm{kb}$ are not prerequisites for knocking in a large $(7.4 \mathrm{~kb})$ fragment into the genomic locus of the gene under study at high efficiency.

\section{Materials and methods}

Mouse ES cell culture

The mouse ES cell line (JM8A3-N1, passage 7) was purchased from the Mouse Biology Program (MBP) at the University of California Davis. ES cells were grown on gelatin-treated cell culture plates with mitomycin-treated feeder cells (PMEF; neo resistant) at $2.5 \times 10^{6}$ cells per $10 \mathrm{~cm}$ plate using Dulbecco's modified Eagle's medium (DMEM), which contains 20\% ES cell qualified fetal bovine serum (FBS), 0.1 mM 2-mercaptoethanol, MEM nonessential amino acids, and $1000 \mathrm{U}$ recombinant murine leukemia inhibitory factor/ $\mathrm{mL}$ (Millipore, Billerica, MA).
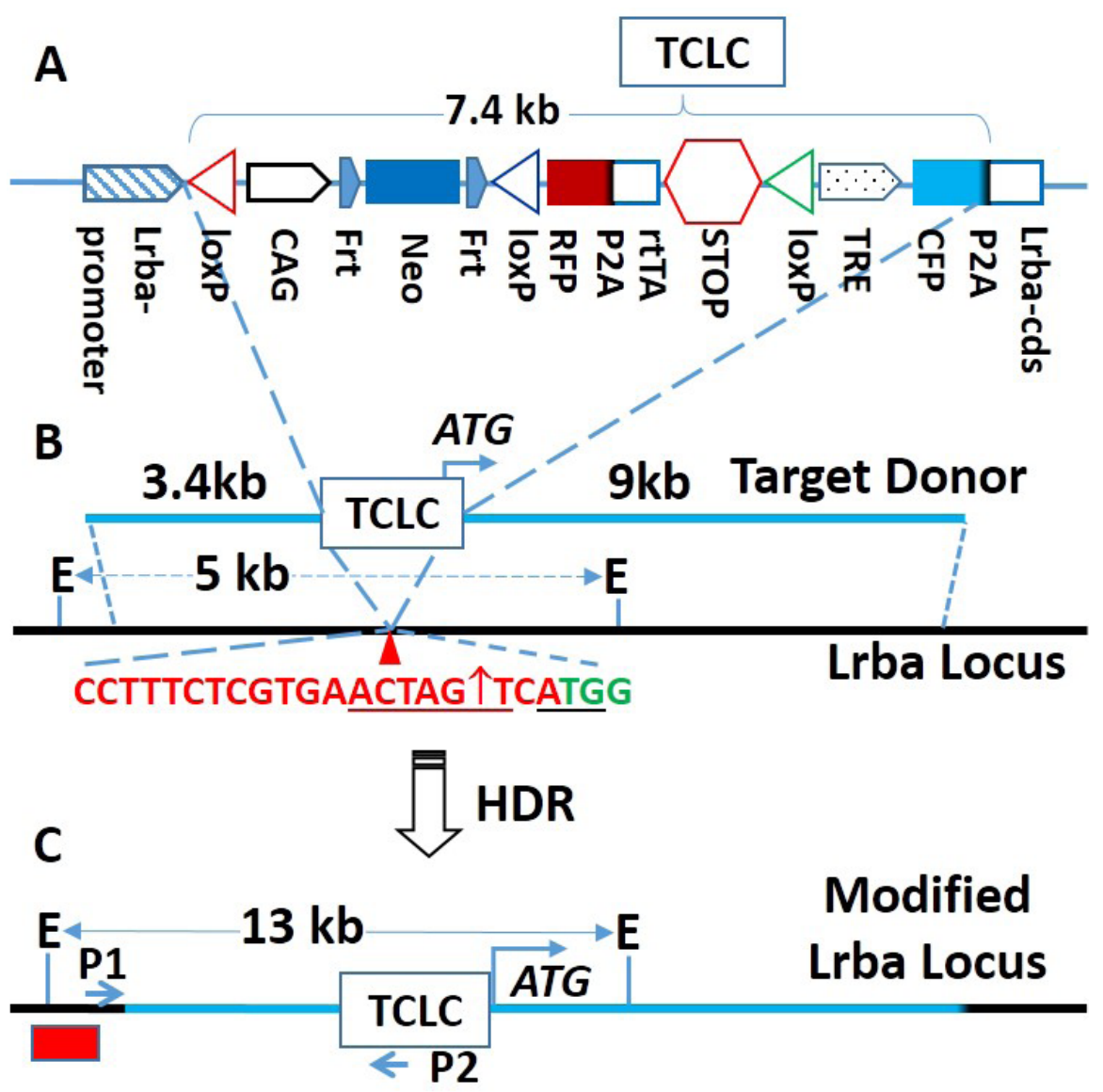

Figure 1. Schematic of CRISPR/HDR targeting strategy. (A) Strategy to knock-in a transcription control and labeling cassette (TCLC) into the Lrba locus in mouse embryonic stem (ES) cells using the CRISPR/homology-directed-repair (HDR) technique. The single guide (sgRNA) sequence is in red, and the protospacer-adjacent motif (PAM) sequence (NGG) is in green. The underlined sequences are the Spel cleavage site and the translation start codon of Lrba. The cleavage site of Cas9 nuclease is indicated by a red arrowhead. (B) The sgRNA will guide the Cas9 nuclease to the target site and cleave the DNA. (C) HDR by the left and right arms (blue) of the target donor will then insert the TCLC into the Lrba locus between the promoter and the Lrba translation start codon (ATG). E: EcoRV site. Red bocks: Southern blotting probes. Triangles: loxP, locus of cross P1 site. STOP: three different polyadenylation signal sites for transcription termination and polyadenylation of mRNA. rtTA: reverse tetracycline controlled transcriptional activator. TRE: tetracycline responsive element.

HDR-mediated gene targeting of mouse ES cells

To generate knockout ES cell clones, ES cells $\left(1 \times 10^{7}\right.$ cells in $0.9 \mathrm{~mL}$ PBS) were electroporated with $15 \mu \mathrm{g}$ of linearized donor vector and $7.5 \mu \mathrm{g}$ of single guide RNA sgRNA vector. The electroporation was performed using the BioRad Gene Pulser II (Bio-Rad Laboratories, Hercules, CA) with the following settings: $250 \mathrm{mV}, 500$ $\mu \mathrm{F}$ capacitance. The ES cells were cultured with $500 \mu \mathrm{g} / \mathrm{mL}$ G418 for 20 days. The G418-resistant colonies were picked and cultured in a 96-well plate until most wells were $\sim 80 \%$ confluent with cells. The cells were then trypsinized and split into 296 -well plates. One plate of ES cells was frozen at $-80^{\circ} \mathrm{C}$, and another plate was used to prepare genomic DNA for PCR screening and confirmation by Southern blotting.

\section{PCR screen of knock-in-positive} ES cell clones

To screen knock-in-positive ES cell clones by PCR, the primers $\mathrm{mLALF}$ and $\mathrm{mLALR}$ (Supplementary Table S1) were designed from the mouse genomic sequence immediately outside of the left homologous arm and the CAG promoter in the target vector, respectively (Figure 1C). The fragment was amplified by PCR in a $20 \mu \mathrm{l}$ reaction mixture composed of $4 \mu \mathrm{l}$ 5x PrimeSTAR (Clontech Laboratories, Mountain View, CA) GXL buffer, $1.6 \mu$ l 2.5 mM dNTP mix, $0.4 \mu \mathrm{l} 10 \mu \mathrm{M}$ forward and reverse primers, $1 \mu \mathrm{l}$ ES cell DNA from the 96-well preparation, $0.4 \mu \mathrm{IDMSO}$, and $0.4 \mu \mathrm{l}$ PrimeSTAR GXL DNA polymerase $(5 \mathrm{U} / \mathrm{ul})$. Sterile distilled water was added to bring the total reaction volume to $20 \mu \mathrm{l}$. The PCR cycling parameters were: $98^{\circ} \mathrm{C}$ 
for 5 min; 5 cycles of $94^{\circ} \mathrm{C}$ for $10 \mathrm{~s}$ and $75^{\circ} \mathrm{C}$ for 3 min $30 \mathrm{~s} ; 5$ cycles of $98^{\circ} \mathrm{C}$ for $10 \mathrm{~s}$ and $72^{\circ} \mathrm{C}$ for $3 \mathrm{~min} 30 \mathrm{~s} ; 26$ to 30 cycles of $98^{\circ} \mathrm{C}$ for $10 \mathrm{~s}$ and $69.4^{\circ} \mathrm{C}$ for 3 min $30 \mathrm{~s}$; and 1 cycle of $72^{\circ} \mathrm{C}$ for $10 \mathrm{~min}$. To detect the presence of the wild-type (WT) allele, the primers LAFWD1 and LAREV1 (Supplementary Table S1) that are located upstream and downstream of the targeting site, respectively, were designed from the mouse genomic sequence. The PCR conditions were the same as the above except for the PCR cycling parameters, which were: $98^{\circ} \mathrm{C}$ for 5 min; 5 cycles of $98^{\circ} \mathrm{C}$ for $10 \mathrm{~s}$ and $72^{\circ} \mathrm{C}$ for $1 \mathrm{~min} ; 5$ cycles of $98^{\circ} \mathrm{C}$ for $10 \mathrm{~s}$ and $69^{\circ} \mathrm{C}$ for $1 \mathrm{~min} ; 30$ cycles of $98^{\circ} \mathrm{C}$ for $10 \mathrm{~s}$ and $66^{\circ} \mathrm{C}$ for 1 min; and 1 cycle of $72^{\circ} \mathrm{C}$ for $10 \mathrm{~min}$. PCR products $(15 \mu \mathrm{l})$ were digested with 5 units of Spel (NEB, Ipswich, MA) and $3 \mu$ of CutSmart buffer in a total volume of $45 \mu \mathrm{l}$ at $37^{\circ} \mathrm{C}$ for $4 \mathrm{~h}$.

We also examined the 3 ' end integrity of the knock-in by detecting the presence of the third loxP sequence using Taqman real-time PCR with primers loxP3F and loxP3R and dual-labeled Taqman probe loxPProbe (Supplementary Table S1). One microliter genomic DNA was used for real-time PCR. All reactions were run on the CFX96 Real-Time System (Bio-Rad Laboratories) in $20 \mu$ l reactions using Premix Ex Taq (Probe qPCR) (Clontech Laboratories) with $10 \mu \mathrm{M}$ primers and probe. The PCR cycling parameters were: $95^{\circ} \mathrm{C}$ for $30 \mathrm{~s}$, followed by 40 cycles of $95^{\circ} \mathrm{C}$ for $5 \mathrm{~s}$ and $60^{\circ} \mathrm{C}$ for $30 \mathrm{~s}$. The baselines and quantification cycle (Cq) were set automatically.

\section{Southern blotting confirmation of} knock-in-positive ES cell clones

Southern blotting was performed using standard methods. The DNA probe was prepared with the PCR DIG Probe Synthesis Kit (Roche Diagnostics, Risch, Switzerland) by using the donor vector as the template with the primers LrbaprbF and LrbaprbR (Table S1). Ten micrograms of genomic DNA were digested with EcoRV overnight and run on a $0.7 \%$ agarose gel. The DNA was transferred to a nylon membrane and hybridized with a digoxigenin-labeled probe. After stringent washing, the membrane was incubated with alkaline phosphataseconjugated anti-digoxigenin antibodies (\#11093274910; Roche Diagnostics). The chemiluminescent detection of positive
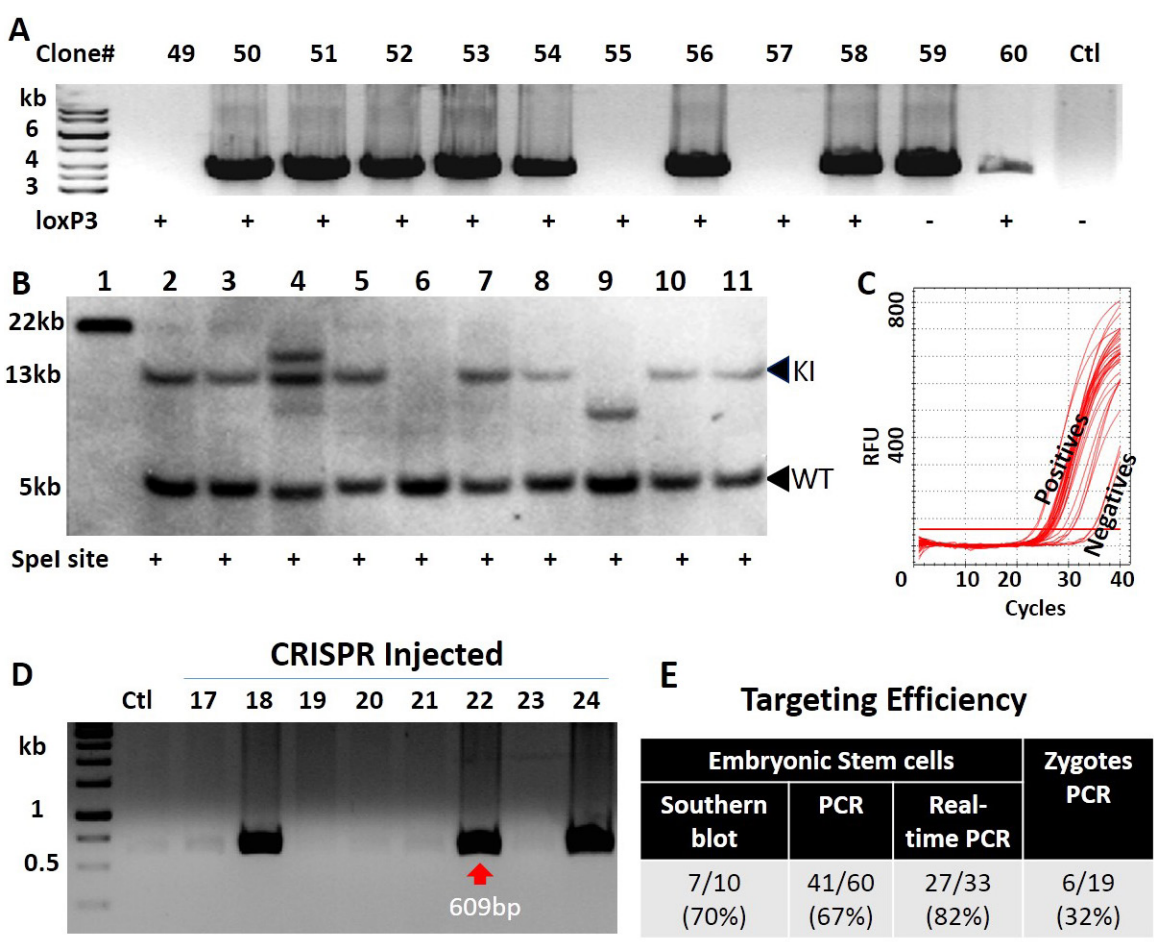

Figure 2. Characterization of mouse embryonic stem (ES) cell clones and blastocysts targeted by CRISPR/homology-directed-repair (HDR). (A) PCR screening for Lrba knock-in-positive ES cell clones. The predicted size of the PCR product is $3.5 \mathrm{~kb}$. Ctl: control. PCR template was from untargeted ES cells. loxP3: the third loxP site. (B) Southern blotting. EcoRV-digested genomic DNA was hybridized with a $5^{\prime}$ external probe with expected fragment sizes of $5 \mathrm{~kb}$ (WT) and $13 \mathrm{~kb}$ (knock-in, KI). Lane 1: linearized donor vector (22 kb, 100 pg) control. Other lanes: G418-positive clones. \#4 and \#9 were incorrectly targeted, and \#6 is WT. The other clones (\#2, 3, 5, 7, 8, 10, and 11) were correctly targeted heterozygotes. (C) The presence of the third loxP site in these ES cell clones was detected by real time PCR using the loxP sequence as a Taqman probe. Some results are shown at the bottom of (A). RFU: relative fluorescence units. Positives: with the presence of the third loxP site; negatives: without the presence of the third loxP site. (D) Nested PCR detection of correctly targeted blastocysts derived from zygotes injected with CRISPR reagents. One quarter of the DNA from a blastocyst was used for the first PCR, as in (A). Ctl: control. PCR template was from an uninjected zygote. (E) Targeting efficiency of CRISPR/HDR in ES cells and zygotes.

signal on the membrane was achieved by incubation with CSPD (a chemiluminescent substrate for alkaline phosphatase) and developed on an X-ray film.

\section{Microinjection of mouse zygotes} with CRISPR reagents

The HDR donor vector was derived from the targeting vector by removing the neomycin expression cassette, which reduced the insert size to $5.8 \mathrm{~kb}$. The Cas9 mRNA was purchased from TriLink BioTechnologies (San Diego, CA). The sgRNA targeting the mouse lipopolysaccharideresponsive vesicle trafficking, beach and anchor containing (Lrba) gene was synthesized in vitro from the PCR products of the pX330-LAsg vector as previously described (16). The 3 CRISPR components were mixed in microinjection buffer (10 mM Tris- $\mathrm{HCl} \mathrm{pH} \mathrm{7.4,} 0.25$ mM EDTA) at concentrations of $5 \mathrm{ng} / \mu \mathrm{l}, 2.5 \mathrm{ng} / \mu \mathrm{l}$, and 10 $\mathrm{ng} / \mu \mathrm{l}$, respectively and were microinjected into the pronucleus at the University of Michigan Transgenic Animal Model Core. Positive control CRISPR reagents were also microinjected into fertilized eggs of the same genetic background under the same conditions and then incubated to follow the in vitro development of microinjected eggs through the blastocyst stage. Uninjected eggs were placed in culture in parallel under the same conditions to determine if medium and incubator conditions were optimal. Animals were housed in an AAALAC accredited facility in accordance with the National Research Council's guide for the care and use of laboratory animals.

\section{Nested PCR detection of targeted blastocysts}

Each blastocyst derived from a zygote injected with CRISPR reagents was put 
Table 1. Fertilized mouse egg development after Cas9 treatments.

\begin{tabular}{|c|c|c|c|c|}
\hline Cas9 DNA construct & Injected eggs & Intact eggs (average \pm SD) & 2-cell eggs (average \pm SD) & Blastocysts (average \pm SD) \\
\hline Lrba/Cas9 diluted* & 60 & $54(90 \pm 0.0 \%)$ & $52(87 \pm 0.0 \%)$ & $37(62 \pm 0.7 \%)$ \\
\hline Lrba/Cas9* & 60 & $38(60 \pm 0.9 \%)$ & $31(74 \pm 3.7 \%)$ & $3(9 \pm 13 \%)$ \\
\hline Controlt & 93 & $82(88 \pm 2.3 \%)$ & $56(60 \pm 1.7 \%)$ & $5(6 \pm 0.2 \%)$ \\
\hline Control + Scr7 & 32 & $25(78 \%)$ & $21(66 \%)$ & $12(38 \%)$ \\
\hline No injection $\dagger$ & N.A. & $60(100 \pm 0.0 \%)$ & $60(100 \pm 0.0 \%)$ & $58(97 \pm 2.9 \%)$ \\
\hline
\end{tabular}

N.A.: not applicable. Two $\left(^{*}\right)$ or three $(\dagger)$ biological replicates. SD: standard error.

into $10 \mu \mathrm{l}$ water and stored at $-80^{\circ} \mathrm{C}$. To extract blastocyst genomic DNA, $10 \mu \mathrm{l}$ $2 \times$ digestion buffer $(100 \mathrm{mM} \mathrm{KCl}, 20 \mathrm{mM}$ Tris- $\mathrm{HCl} \mathrm{pH}$ 9.0, 0.2\% Triton $\mathrm{X}-100$, and $0.8 \mathrm{mg} / \mathrm{mL}$ proteinase $\mathrm{K}$ ) were added into each tube containing 1 blastocyst and incubated at $55^{\circ} \mathrm{C}$ overnight, followed by incubation at $94^{\circ} \mathrm{C}$ for $10 \mathrm{~min}$ to inactivate the proteinase K. Five microliters of the blastocyst DNA preparation were used as PCR template for the first PCR reaction using the same conditions and parameters as the PCR screening of knock-in-positive ES cell clones, with the exception that the number of cycles in the third phase of the touchdown PCR program was changed to 20. The second (nested) PCR reactions were conducted as follows: the nested PCR primers are LAFWD1 and FL30R (Supplementary Table S1), and the PCR conditions were the same as the above except for the cycling parameters, which were: $98^{\circ} \mathrm{C}$ for 5 min; 5 cycles of $98^{\circ} \mathrm{C}$ for $10 \mathrm{~s}$ and $69.5^{\circ} \mathrm{C}$ for $1 \mathrm{~min} ; 5$ cycles of $98^{\circ} \mathrm{C}$ for $10 \mathrm{~s}$ and $66.5^{\circ} \mathrm{C}$ for $1 \mathrm{~min}$; 28 cycles of $98^{\circ} \mathrm{C}$ for $10 \mathrm{~s}$ and $63.5^{\circ} \mathrm{C}$ for $1 \mathrm{~min}$; and 1 cycle of $72^{\circ} \mathrm{C}$ for $10 \mathrm{~min}$.

\section{Results and discussion}

We sought to integrate a $7.4 \mathrm{~kb}$ transcription control and labeling cassette (TCLC) at the Lrba genomic locus in mice (Figure 1). A donor Lrba vector was constructed from a BAC subclone, had homologous arms of $3.4 \mathrm{~kb}$ and $9 \mathrm{~kb}$ flanking the TCLC, and was characterized by restriction digestion and then confirmed by sequencing (Figure 1A, Supplementary Figure S1).

We found that $67 \%$ of G418-positive ES cell clones were correctly targeted for the $5^{\prime} \mathrm{HR}$ (Figure 2A), while more than $82 \%$ of G418-positive clones also had the third loxP sequence (loxP3, Figure 2C and bottom of Figure 2A), the presence of which may be an indication of the integrity of the $3^{\prime}$ end of the insert. The clones that were positive for both 5 '-recombination and the third loxP sequence have likely been correctly targeted. Since PCR approaches cannot detect concurrent random integration, Southern blotting was used to confirm the presence of the TCLC. We found that $70 \%$ of clones were correctly targeted based on the Southern blot (Figure 2B).

This high CRISPR/HDR-mediated knock-in efficiency obtained in ES cells prompted us to test whether similar efficiencies could be obtained in mouse zygotes. The CRISPR reagents injected at the regular concentrations (8) interfered with the normal development of zygotes. Only 9\% of zygotes injected with the CRISPR reagents developed into blastocysts (Table 1). To reduce this toxicity, the reagents were diluted 4 -fold. The diluted reagents produced the best results when compared with the positive controls with or without the DNA ligase IV inhibitor Scr7, which inhibits NHEJ (Table 1). Nested-PCR genotyping revealed that the targeting efficiency for mouse zygotes was 32\% (Figure 1D). This frequency would have been higher if the regular concentrations of CRISPR reagents had been used. High efficiency of HDR-mediated knock-in in mice is expected as the CRISPR knockout efficiencies are almost the same in ES cells and mice (4). The advantages of using lower concentrations of CRISPR reagents may include lower toxicity, lower off-target rates, and lower cost. Evidence of genome editing in blastocysts indicated that genome-edited mice could be produced. We then injected 311 embryos with the diluted CRISPR reagents and obtained a survival rate of $82 \%$ for the blastocysts, which were transferred to pseudopregnant recipients. We obtained a $34 \%$ birth rate (86 pups). The high survival rate of blastocysts and the high birth rate support the use of low concentrations of CRISPR reagents.

The high efficiency of CRISPR/HDR (Figure 1E) without NHEJ inhibitors suggests that long homologous arms (3.2 and $7.6 \mathrm{~kb}$ ) (Supplementary Table S2), which are much longer than the recommended $800 \mathrm{bp}$ (8), are required to obtain high knock-in efficiency (17). The frequency of traditional gene targeting is roughly proportional to the extent of homology shared by the transgene and its target locus (18). For example, a 40-fold increase in the rate of targeting has been observed with an increase in homology from $4 \mathrm{~kb}$ to $9.1 \mathrm{~kb}$ (19). A 25 -fold increase was seen over the range of $2.5 \mathrm{~kb}$ to $9.5 \mathrm{~kb}$ of homology (20). We thus conducted the parallel comparison of targeting efficiency of two targeting vectors with different lengths of homologous arms flanking the same insert (Supplementary Table S2). Although there is total of $\sim 7 \mathrm{~kb}$ difference of the homologous sequences between the 2 targeting vectors, the results show that there is no obvious difference in targeting efficiency between the 2 targeting vectors (Figure 3A). This indicates that the targeting efficiency of CRISPR/HDR is not dependent on the lengths of each homologous arm in the range of $1.7 \mathrm{~kb}$ to $7.6 \mathrm{~kb}$, and that increasing the length of each homologous arm in this range may not increase the CRISPR/HDR targeting efficiency as it does for traditional gene targeting. Our results also show that a $\sim 2$ kb homologous arm at each end should be sufficient for CRISPR/HDR, and it can be easily obtained by PCR amplification, thus making it easier to construct a targeting vector. Although extremely short (50 bp) homologous arms of double-stranded DNA can mediate HDR at 5\%-10\% efficiency, they may not efficiently mediate HDR of a larger insert such as the $7.4 \mathrm{~kb}$ fragment used in this study (21). Indeed, increasing the insert length from $99 \mathrm{bp}$ to $720 \mathrm{bp}$ considerably reduced targeting efficiency (9-fold), but that can be compensated for by increasing the homology arm size (22). Increasing the lengths of homologous arms in the range of $50 \mathrm{bp}$ to $2 \mathrm{~kb}$ likely increases the targeting efficiency.

While high CRISPR/HDR gene targeting efficiency was obtained for both ES cells and zygotes, no biallelic knock-ins were detected by Southern blotting and PCR screening in ES cells. It is possible that the mutated alleles may have NHEJ-mediated small insertion/deletion (indel) mutations that cannot be distinguished from the WT 
alleles by the two methods used above. These mutations would destroy the Spel site (Figure 1B) in the Cas9/sgRNA recognition site, since the Cas9/sgRNA complex cleaves 3-8 nucleotides upstream of the protospacer adjacent motif (PAM) (23-25), and this Spel site is located just within the cleavage range. Although the feeder cells contribute to the PCR products, their numbers in each culture well were 20 times less than that of ES cells. Therefore, the PCR products should mainly originate from the ES cells. Spel digestion can thus be used to detect NHEJ-induced mutations. In a CRISPR/NHEJ experiment, Spel can digest the PCR product (713 bp) from the WT allele, producing $527 \mathrm{bp}$ and $186 \mathrm{bp}$ fragments, but it cannot cut the CRISPR-mutated allele as the Spel site would be destroyed by the CRISPR mutation (Figure 3B). However, in the CRISPR/HDR experiments, all PCR products were digested by Spel (Figure $3 \mathrm{C})$; there was no uncleaved band for any clone among the 41 targeted clones and the 19 non-targeted clones. These results indicate that all of these clones have at least one WT allele, but there are

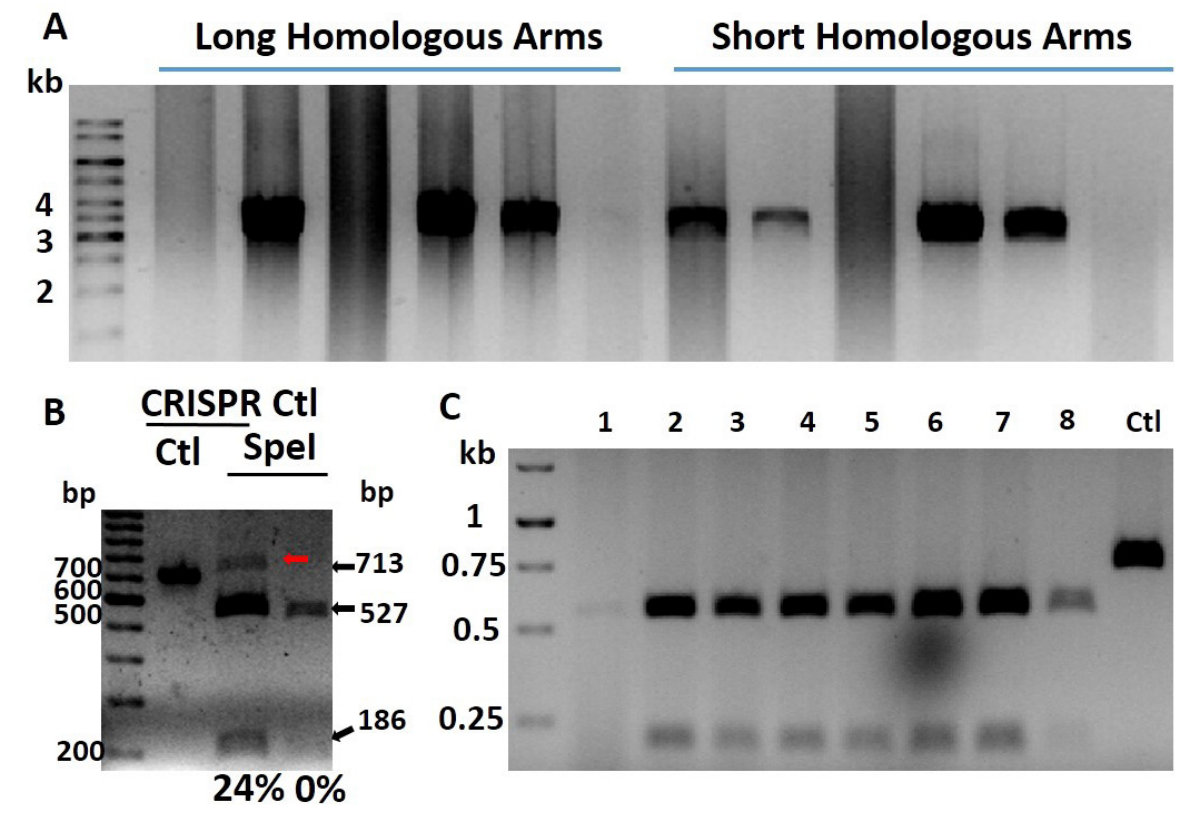

Figure 3. CRISPR/homology-directed-repair (HDR) and CRISPR/nonhomologous end-joining (NHEJ) pathways. (A) Comparison of the targeting efficiencies of two targeting vectors with different lengths of homologous arms flanking the same insert (Supplementary Table S2). Thirty G418-positive ES cell clones were picked and subjected to PCR screening of Lrba knock-in-positive ES cell clones. The predicted size of the PCR product was $3.5 \mathrm{~kb}$. Representative results are shown. (B) Spel digestion of PCR products using genomic DNA from ES cells transfected with a Lrba sgRNA/ Cas9 vector. Spel digestion of the 713 bp PCR product produces 527 bp and 186 bp fragments in WT samples but cannot cut the CRISPR mutated allele (red arrow) as the Spel site was destroyed by CRISPR. The percentages at the bottom are the estimated targeting efficiencies. (C) Detection of CRISPR/NHEJ-mediated mutations in ES cell clones by Spel digestion. PCR products were obtained from 56 of 60 ES cell clones with either targeted or random integration and were subjected to Spel digestion. Ctl: no Spel, undigested PCR product. Representative results are shown.

no mutated alleles resulting from CRISPR/ NHEJ, while this Cas9/sgRNA system can completely cleave its substrate in vitro (16) and successfully mutated the target site in ES cells at the absence of a donor vector (Figure 3B).

This is a surprising result given that the usually higher than that of $(4,8)$. NHEJ occurs throughout the cell cycle, while HDR occurs only during the $S$ and $G 2$ phases (13). These two repair ways seem to compete for DSBs, and efficiency of $\operatorname{HDR}(13,14)$. On the other hand, the CRISPR/Cas9 cleavage site is complete repair of a DB by NHEJ. This cycle of cleavage/NHEJ-repair may be repeated multiple times until the DSB is repaired with a mutation. However, once a DSB is repaired by HDR and a donor DNA fragment is inserted, the CRISPR/Cas9 cleavage site is destroyed and cannot be cleaved again by CRISPR/Cas9 (26). In this sense, CRISPR/HDR is more efficient than CRISPR/NHEJ, which may require multiple rounds of cleavage/repair. In

\begin{tabular}{l|l|l|l} 
Vol. $59 \mid$ No. $4 \mid 2015$ & 207
\end{tabular} $\because \because \because$ KAPABIOSYSTEMS

Introducing KAPA PROBE FORCE

Direct $\mathrm{qPCR}$ analysis without sample purification

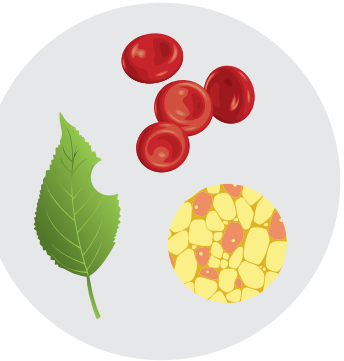

Direct analysis from crude extracts

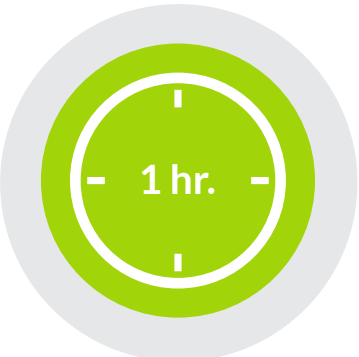

Streamlined workflows

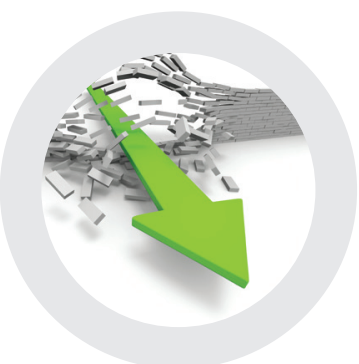

Superior inhibitor tolerance

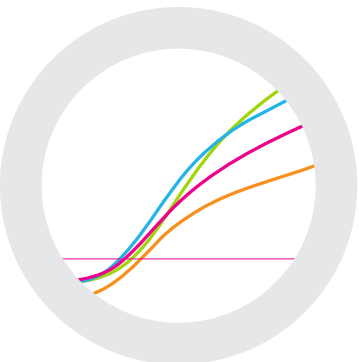

Multiplex compatibility

\section{Visit}

kapabiosystems.com/probeforce to request a trial kit 
this study, it is possible that the NHEJ repairs almost all of the DSB sites at this genomic site without mutation, or that the NHEJ-mediated mutations are inhibited by some unknown mechanisms. As for no biallelic knock-ins being detected, it is likely that the double knock-in is lethal to the cells because knockdown of Lrba induces apoptosis (27). Since whether cells choose NHEJ or HDR is fundamental to CRISPR/Cas9 gene targeting, further investigation of these surprising results is warranted.

Another intriguing observation is that most of the G418-positive clones resulted from HR. This result is in sharp contrast to the traditional targeting, where most of the G418-positive clones result from random integration. The frequency of random integration is typically 1000 times more than that of targeted integration (18). These results indicate that by increasing DNA DSBs at the correct site, CRISPR favors on-target integration as opposed to random integration.

We have demonstrated that $(i)$ the targeting efficiency of CRISPR/HDR without using any NHEJ inhibitors is equivalent to that of CRISPR/HDR with NHEJ inhibitors; (ii) CRISPR/NHEJ is not detectable; (iii) biallelic knock-in is not detectable; (iv) left and right homologous arms longer than 2.1 and $1.7 \mathrm{~kb}$, respectively, do not increase HDR efficiency; ( $v$ ) one fourth of the regular concentrations of CRISPR reagents can be wused to knock in a large DNA fragment into the genome of mouse zygotes at high efficiency with low toxicity; and (vi) large DNA fragments, 7.4 and $5.8 \mathrm{~kb}$, can be knocked in to the genomes of ES cells and zygotes, respectively. These results should inspire interest in further study of the mechanisms of high CRISPR targeting efficiency, as well as provide an example of how to obtain high efficiency gene knock-in results with large DNA fragments using CRISPR/HDR without the need for NHEJ inhibitors.

\section{Author contributions}

J.W. contributed to the conception of the study, writing and editing of the manuscript, and analysis of the experiments. B.W., K.L., A.W., and M.R. contributed to the execution of the experiments. T.S. contributed to the injection and culture of mouse zygotes.

\section{Acknowledgments}

This work was supported by the Joy McCann Culverhouse Endowment to the Division of Allergy, Department of Internal Medicine, and the University of Michigan Transgenic Animal Model Core.

\section{Competing interests}

The authors declare no competing interests.

\section{References}

1. Wiedenheft, B., S.H. Sternberg, and J.A. Doudna. 2012. RNA-guided genetic silencing systems in bacteria and archaea. Nature 482:331338.

2. Brown, A.J., D.A. Fisher, E. Kouranova, A. McCoy, K. Forbes, Y. Wu, R. Henry, D. Ji, et al. 2013. Whole-rat conditional gene knockout via genome editing. Nat. Methods 10:638-640.

3. Cong, L., F.A. Ran, D. Cox, S. Lin, R. Barretto, N. Habib, P.D. Hsu, X. Wu, et al. 2013. Multiplex genome engineering using CRISPR/Cas systems. Science 339:819-823.

4. Wang, H., H. Yang, C.S. Shivalila, M.M. Dawlaty, A.W. Cheng, F. Zhang, and R. Jaenisch. 2013. One-Step Generation of Mice Carrying Mutations in Multiple Genes by CRISPR/Cas-Mediated Genome Engineering. Cell 153:910-918.

5. Lieber, M.R. 2010. The mechanism of doublestrand DNA break repair by the nonhomologous DNA end-joining pathway. Annu. Rev. Biochem. 79:181-211.

6. Frit, P., N. Barboule, Y. Yuan, D. Gomez, and P. Calsou. 2014. Alternative end-joining pathway(s): bricolage at DNA breaks. DNA Repair (Amst.) 17:81-97.

7. Ding, Q., S.N. Regan, Y. Xia, L.A. Oostrom, C.A. Cowan, and K. Musunuru. 2013. Enhanced efficiency of human pluripotent stem cell genome editing through replacing TALENs with CRISPRs. Cell Stem Cell 12:393-394.

8. Yang, H., H. Wang, C.S. Shivalila, A.W. Cheng, L. Shi, and R. Jaenisch. 2013. One-step generation of mice carrying reporter and conditional alleles by CRISPR/Cas-mediated genome engineering. Cell 154:1370-1379.

9. Zhou, J., J. Wang, B. Shen, L. Chen, Y. Su, J. Yang, W. Zhang, X. Tian, and X. Huang. 2014. Dual sgRNAs facilitate CRISPR/Cas9-mediated mouse genome targeting. FEBS J. 281:1717-1725.

10. Fujii, W., K. Kawasaki, K. Sugiura, and K. Naito. 2013. Efficient generation of large-scale genomemodified mice using gRNA and CAS9 endonuclease. Nucleic Acids Res. 41:e187.

11. Bollag, R.J., A.S. Waldman, and R.M. Liskay. 1989. Homologous recombination in mammalian cells. Annu. Rev. Genet. 23:199-225.

12. Donoho, G., M. Jasin, and P. Berg. 1998. Analysis of gene targeting and intrachromosomal homologous recombination stimulated by genomic double-strand breaks in mouse embryonic stem cells. Mol. Cell. Biol. 18:4070-4078.

13. Maruyama, T., S.K. Dougan, M.C. Truttmann, A.M. Bilate, J.R. Ingram, and H.L. Ploegh. 2015. Increasing the efficiency of precise genome editing with CRISPR-Cas9 by inhibition of nonhomologous end joining. Nat. Biotechnol. 33:538-542.
14. Chu, V.T., T. Weber, B. Wefers, W. Wurst, S. Sander, K. Rajewsky, and R. Kuhn. 2015. Increasing the efficiency of homology-directed repair for CRISPR-Cas9-induced precise gene editing in mammalian cells. Nat. Biotechnol. 33:543-548.

15. Xue, W., S. Chen, H. Yin, T. Tammela, T. Papagiannakopoulos, N.S. Joshi, W. Cai, G. Yang, et al. 2014. CRISPR-mediated direct mutation of cancer genes in the mouse liver. Nature 514:380-384.

16. Wang, J.W., A. Wang, K. Li, B. Wang, S. Jin, M. Reiser, and R.F. Lockey. 2015. CRISPR Cas9 nuclease cleavage combined with Gibson assembly for seamless cloning. Biotechniques 58:161-170

17. Song, H., S.K. Chung, and Y. Xu. 2010. Modeling disease in human ESCs using an efficient BAC-based homologous recombination system. Cell Stem Cell 6:80-89.

18. Smith, K. 2001. Theoretical mechanisms in targeted and random integration of transgene DNA. Reprod. Nutr. Dev. 41:465-485.

19. Thomas, K.R. and M.R. Capecchi. 1987. Sitedirected mutagenesis by gene targeting in mouse embryo-derived stem cells. Cell 51:503-512.

20. Shulman, M.J., L. Nissen, and C. Collins. 1990. Homologous recombination in hybridoma cells: dependence on time and fragment length. Mol. Cell. Biol. 10:4466-4472.

21. Orlando, S.J., Y. Santiago, R.C. DeKelver, Y. Freyvert, E.A. Boydston, E.A. Moehle, V.M. Choi, S.M. Gopalan, et al. 2010. Zinc-finger nuclease-driven targeted integration into Mamm. Genomes using donors with limited chromosomal homology. Nucleic Acids Res. 38:e152.

22. Li, K., G. Wang, T. Andersen, P. Zhou, and W.T. $\mathrm{Pu}$. 2014. Optimization of genome engineering approaches with the CRISPR/Cas9 system. PLoS ONE 9:e105779.

23. Jinek, M., K. Chylinski, I. Fonfara, M. Hauer, J.A. Doudna, and E. Charpentier. 2012. A programmable dual-RNA-guided DNA endonuclease in adaptive bacterial immunity. Science 337:816-821.

24. Li, Y., A.I. Park, H. Mou, C. Colpan, A. Bizhanova, E. Akama-Garren, N. Joshi, E.A. Hendrickson, et al. 2015. A versatile reporter system for CRISPR-mediated chromosomal rearrangements. Genome Biol. 16:111.

25. Doudna, J.A. and E. Charpentier. 2014. Genome editing. The new frontier of genome engineering with CRISPR-Cas9. Science 346:1258096.

26. Singh, P., J.C. Schimenti, and E. Bolcun-Filas. 2015. A mouse geneticist's practical guide to CRISPR applications. Genetics 199:1-15.

27. Wang, J.W., J.J. Gamsby, S.L. Highfill, L.B. Mora, G.C. Bloom, T.J. Yeatman, T.C. Pan, A.L. Ramne, et al. 2004. Deregulated expression of LRBA facilitates cancer cell growth. Oncogene 23:4089-4097.

Received 30 March 2015; accepted 27 July 2015.

Address correspondence to Jia-Wang Wang, Division of Allergy and Immunology, Department of Internal Medicine, University of South Florida Morsani College of Medicine, Tampa, FL. E-mail: jwang@health.usf.edu

To purchase reprints of this article, contact: biotechniques@fosterprinting.com 